IB Lawrence Berkeley Laboratory

WNINERSTTYFOALIORNIA

Accelerator \& Fision.
Research Division.

LBL -18963

DE85 017642

Presentea at the 1985 partlal a Acelerator conferences thI May $13-16,1985$

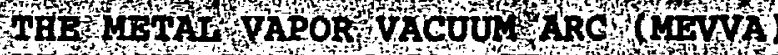

HIGH CORRENT ION SOORCE

\title{
I.G. Brown
}

May 1985
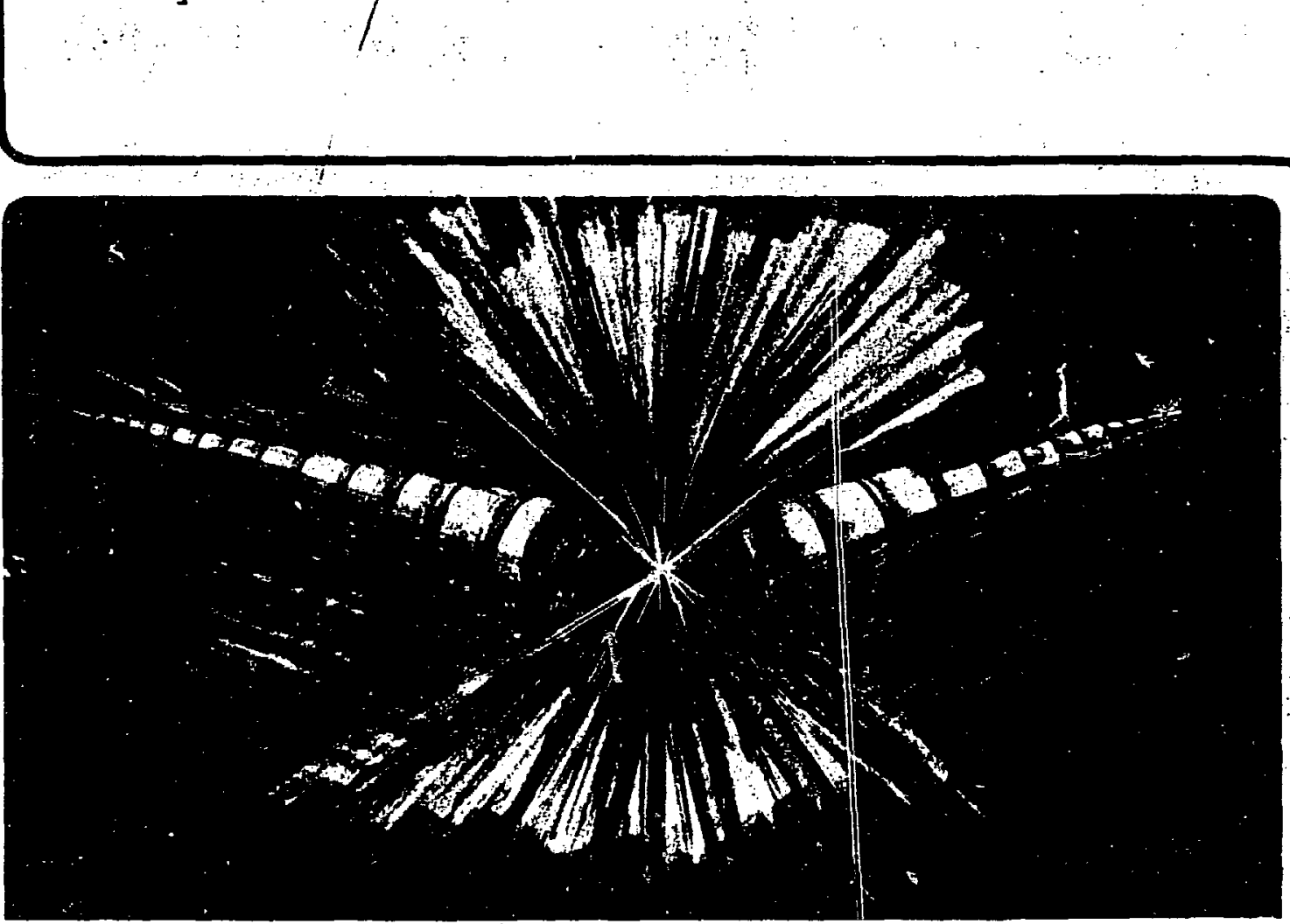

Prepared for the U.S. Department of Energy under Contract DE-AC03-76SF00098 
This report was done with support from the Department of Energy. Any conclusions or opinions expressed in this report represent solety those of the author(s) and not necessarily those of The Regents of the University of California, the Lawrence Berkeley Laboratory or the Department of Energy.

Reference to a company or product name does not imply approval or recommendation of the product by the University of California or the U.S. Department of Energy to the exclusion of others that may be suitable. 


\author{
Ian G. Brown \\ Lawrence Berkeley Laboratory \\ University of California \\ Berkeley, CA 94720
}

\section{ABSTRACT}

An ion source is described in which a methl vepor vacuum arc is used to create the plasma from which the ions a re extracted. Beams of a variety of ions have been produced, ranging from lithium up to uranium. At an extraction voltage of $25 \mathrm{kV}$ we've measured an ion beam current of over 1 Ampere, with over 550 ema of the beam in an emittance of $0.07 \pi c m$. mradians (normalized). The ion charge state distribution varies with cathode material and with arc power; for : Iranium a typical distribution is peaked at $\mathrm{U}^{5+}$, with up to 408 of the beam current in this charge stute.

\section{INTRODUCTION}

Progress in the development of high current ion sources has been significant in the last decade. The requirements of the magnetic fusion research progrum have fostered the development of neutral besm sources cupable of delivering beams of hydrogen isotopes having equivalent currents up to the 100 Amp range $\{1,2\}$. The heavy ion fusion research program has seen the development of sub-microsecond beams with current approuching 1 MA [3-5]. The production of long pulse or dc ion beams from solids has not witnessed similar progresa however. These kinds of beams have to-dute been obtained by employing vaporization of the solid $|6-9|$, or surface ionization $[6,10]$, or sputtering $(6,11,12\}$. Beams of uranium ions have been produced by a PIG source using sputtering, of intensity up to several tens of milliamperes [11].

The source that we've developed makes use of a dense plasma created directly from the solid material, us the medium from which ions are extracted. The plasma is created using a metal vapor arc discharge in vacuum. Extraction of ions from the plasma is accomplished using an accel-decel, multi-aperture grid system, and un intense ion beam composed of the cathode material is thereby produced. The application of metal vapor arcs to the production of beams of metal ions has been recognized recently by several workers $[13,14]$. This paper describes the embodiment of the concept that we've developed at LBI. Distinguishing characteristics of our source include a high quality beam and a longpulse or dc extraction system.

\section{DESCRIPTION OP THE SOURCE}

The MEVVA ion source is comprised of a metal vapor vacuum arc plasma source, a drift or plusm shaping region, and a set of grida for ion extruction. The structure is housed within a simple vacuum chrmber having a base pressure in the $10^{-6}$ Torr rnnge. A schemutic of the source is shown in Figure 1 .

- Work supported by the Director, Ofrice of Energy Research, Office of High Energy und Nuclear Physies, Nuclegr Science Division, U.S. Department of Enerty, winder contract number DE-AC03-76SFU0098.

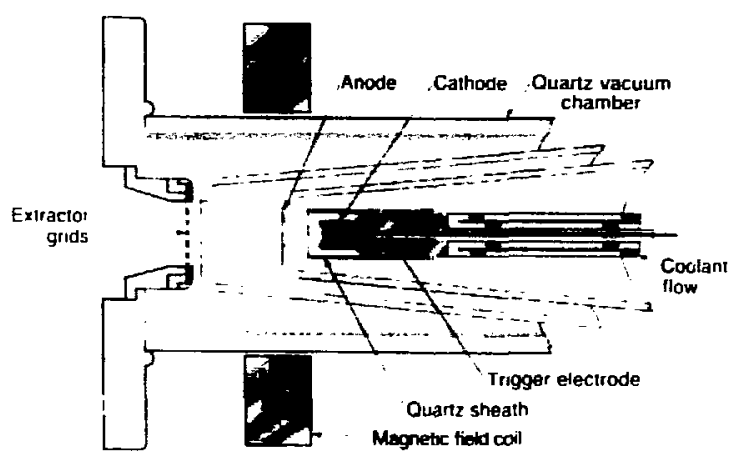

Figure 1 . MEVya ion source. Uutline of the arc and extractor regions.

The metal vapor arc is a plasma phenomenon tha! has been well investiguted for many yeurs $(15,16)$; it is a field of reseurch that has largely been the domain of the high current switching community. We've drawn upon this body of work, and most eypecially from the work of (filmour and lockwoud [17] who have described a configuration which is psrticularly well-suited to the requirements of an ion source. In the metal vapor arc discharge. material is vaporized and ionized ut the cathode surfuce through the formation of 'cathode spots' - minute regions of intense current concentration (though! to be of order $10^{6} \mathrm{Amps} / \mathrm{cm}^{2}$ or greuter). Many auch cathode spots participate in a typical vacuum arc digrharge, the net result of which is the formution of a d:nse plasma of cathode muterial. This quasineutral plasma plumes away from the cathode townrd the anode. A portion of the plusma plume impinges upon the unode, completing the electrical circuit und allowing the arc to persist for as long as the external current drive is maintained. The central part of the plasma plume, however, is allowed to flow through a central hole in the anode, and it is this component of the plasma that forms the medium from which ions are extructed. The plasma plume drifis through the post-unode region to the set of sridu that comprise the ion extractur.

The ion extraction system consists of a set of three grids, each of which is an array of ubout 100 or more small holes of dianeter about $1 \mathrm{~mm}$ or 80 , with un array diameter of $2 \mathrm{~cm}$. The first grid (closest to the anode) is connected to the anode via $500 \mathrm{Ohm}$ resistor; the necond (middle) grid it the electron suppressor and is held at about $-1 \mathrm{kV}$; the third (outerinost) grid is tied to ground. The arc (cathode-snode eircuit) and the first grid are flosted to extractor potential, which for our work to-date has been in the runge 10 - $25 \mathrm{kV}$. Thus the ion accelerating voltage appears across the gap between grids one and two. This kind of grid system is well-understood and has been descrited by a number of authors $\{18,19]$. 
The arc is initiated by a trigger êlect rode located coaxially within the cathode and separated from it by a thin alumina sheath. A short pulse of aeveral micraseconds duration and of amplitude $10-20 \mathrm{kV}$ causes sufficient initial ionizution 1 , ullow the main arc to grow. Various other means of triggering the arc, apart from this coaxial trigger electrode configuration, are possible and we are currently investigating elternatives.

A magnetic field coil located at the anode plane serves to establish a small field throughout the plasma region, of strength up to a few hundred Gauss. The plasma plume is thereby guided maximally through the anode hole. This feature also adds an additional control over the denuity and radial proble of the plauma plume ut the extructor, and thus allows for optimization of the extraction optics and production of a high quality beam. It is also possible that the magnetic field helps to suppress the growth of mhd instabilities in the arc plasma because of the favorable field curvature 120,211 ; this would in turn produce a more quiescent beam than might otherwise occur. None-the-less, the magnetic field is not an essential feature of the source, ard we've often operated with the mognet off.

We've constructed and tested several versions of the MEVVA ion source. Figure 1 is a schematic outline of the are and extractor region of MEVVA II, that embodiment with which we've carried out most of our work. A photograph of the disassembled MEVVA 11 source is shown in Figure 2. We've also made a smaller version, MEVVA IIl, and a photograph of this source is shown in Figure 3.

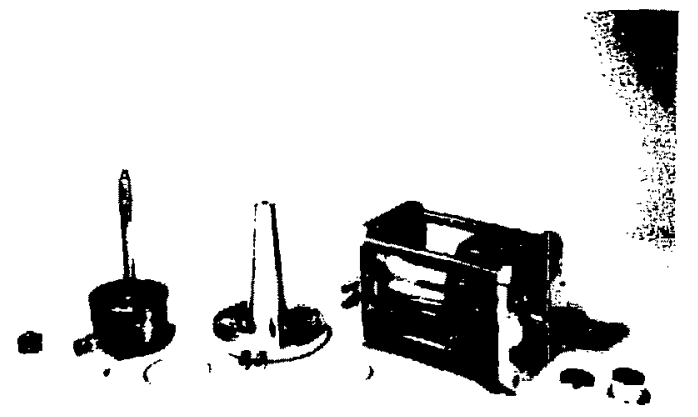

Figure 2. Photograph of the disassembled IFVVA II source.

\section{SOURCE PERPORMANCE}

For accelerator application at Lawrence Berkeley Laboratory we're mainly interested in producing intense uranium beams in the SuperHILAC ( $8.5 \mathrm{MeV} / \mathrm{amu})$ and the Bevalac $[22,23,24\}(\approx 1 \mathrm{GeV} / \mathrm{amu})$, and thus we've run the MFVVA source using a cathode fabricuted of depleted urunium. For simplicity in handling, however, most of our development work was done using tantalum. The complete range of cuthode muterials that we've tried includes: lithium, curbon, aluminum, silicon. titanium, iron, niobium, tantalum, gold, lead, uranium, and Janthunum hexaboride. In all cases, we've extracted beam and sampled the ion charge state distribution (CSD) using * time-of-flight diagnostic.

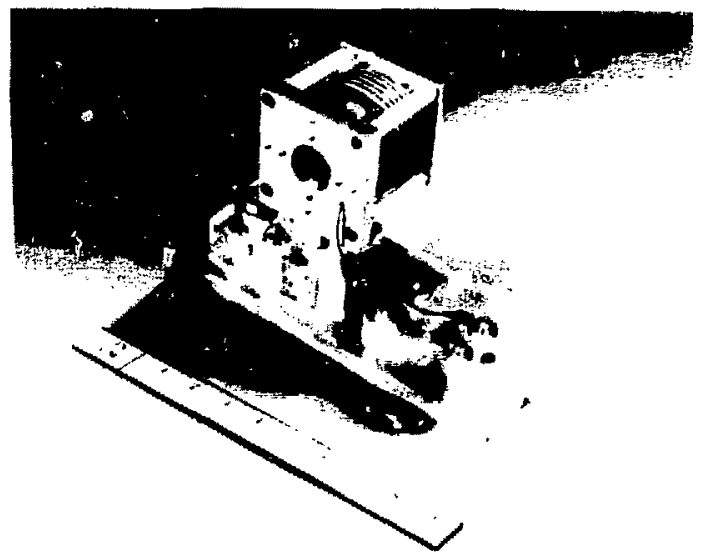

Fi:sure 3. Photograph of MEVVA 111.

In these tesis the arc was driven by a 300 microseco:ad, 6-section, LC pulse line of impedance 0.5 Ohms with a Gibbs section on the front end to sharper, the rige-tine. We've also run with an arc pulse length as long as 3 milliseconds, and we've operated the arc a)one, without extraction of beam, for an on-time of 20 secqnds. Repetition rate was typically about 2 pulses per second; the maximum rate of about 10 pps was set by the electronics available to us. The main concern to be addressed at high duty cycle or dc is the provision of adequate cooling.

Some conditioning of a newly-assembled source is needed. As is usual for high voltage, high current ion sources it is necessary to "bake in" the extractor gridy in a preliminary conditioning run in order to obtain full voltage holding capability. The source is run repetitively while slowly increasing the arc current and extractor voltage. Onçe the grids have been conditioned in this way they'll hold full voltage indefinitely, requiring only minimal re-conditioning after exposure to atmosphere or handling. The cathode itgelf also needs a similar bake-in before a clean spectrum can be obtained. Interestingly, we observe that the small fraction of impurity ions present in the beam apectrum is further reduced as the pulse repetition rate is increased, presumubly because of the reduction in cathode surface impurity level build-up, and perhaps also due to pumping of the arc environment by freshly deposited metal.

A bright and well collimated plasma plume is generuted by that part of the arc plasma that flows through the anode hole. Figure 4 is a photograph of a uranium plasma plume in the MEVVA 1 source.

Note that the apparent blow-up of the beam is not a spвce charge effect since the plume is a quasi-neutral plasma, but is due to the magnetic ducting of the plusma; the plasma radius obeys the fux conservation rule $\Delta r^{2}=$ constant. A thin aluminum foil was loceted so as to collect the mass of tantalum transferred by the plume. The mass of the foil was determined before and ufter a series of several hundred shots with an ar: current of 440 Amps. The specific mass transfer was found to be $4 \times 10^{-9} \mathrm{Kgm} /$ Coulomb of arc current. Note that this refers not to the total mass evolved froin the cathodis, but just to that component that etreams 
through the anode hole and is thus available to the extraction $\mathrm{g}$ rids for the creation of beam. This value is only about 58 of the total cathode mass evolution, in the form of ions, as estimated by previuus workera $[17,25]$, and might be tuken as an indication of the effeiency with which the plume transfers mass (and charge) out of the arc for our geometry. One might thus speculate that an improvement in this efticiency might be possible, of up to about an order of mygnitude, by judicious choice of geometry.

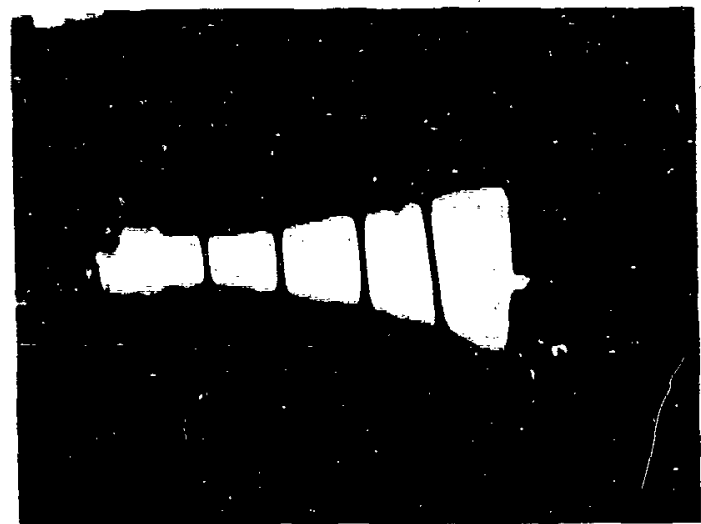

Figure 4. Uranium plasma plume streaming through the anode hole (left) and expanding along the magnetic field en route to the extractur (right). MEVVA 1 .

We have uged a gridded electrostatic energy analyzer to measure the longitudinal ion temperuture of the plusma in the plume. We obtained a value of $15 \mathrm{eV}$, and this may be taken a a minimum value of the energy spread of the extructed ion beam also. Further, if we bssume that the transverse ion temperature equals the longitudinal temperature (equilibration within the cathode spot plasma), then we can can estimate a lower bound for the angular divergence of the extracted beam:

$$
\theta_{\min }=v_{i L} / v_{i l}=\left(T_{u} / \overline{Q V}_{\text {oxt }}\right)^{l / 2}
$$

which for $\bar{Q}=3$ for tantalum and an extraction voltage of $20 \mathrm{kV}$ gives $\theta_{\mathrm{min}}=0.9^{\circ}$, or $\mathrm{an}$ emittance $\mathrm{c}_{\mathrm{min}}=$ 0.013 n cm.mrad.(normalized). This is the minimum emittance obtainable, in the case that the extraction is ideal.

The current in the extracted beam has been measured using several different kinds of Faraday cups and calorimeters. These different techniques all agree with an uncertainty of no more than \pm 208 . We have not measured beam current for all cathode materials and ull operating conditions, but we have a fair sampling. The highest current that we've measured was 1.1 Amps; this was a tantalum beam (all charge stutes) extracted at 25 $k V$. We've also measured 0.5 Amps of niobium at $14 \mathrm{kV}$ and 0.8 - 1.0 Amps of lithium at $17 \mathrm{kV}$. (These duta were taken under. different operating conditions, and should not be compared quantitatively to each other).

Arother important diagnostic that we've employed is a 16-cup beam profile monitor [26]. An example of output from this disgnostic is shown in Figure 5 . The smooth curve is a fitted Gaussian, from which the beam parameters were derived.

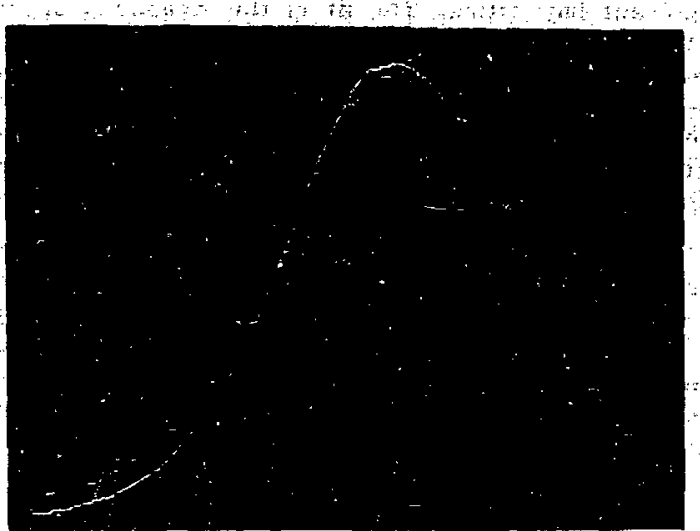

Figure 5. lkadinl current profile вs nensured-by 16-cup prohile monitor, with fitted Guussian.

We have yet to carry out a detailed study of beam emittance using the traditional scanning technique $|6|$. i- 'uve however used beam profile measurements and the known geometry to obtuin a reasonable estimate of emittance; on-line computer analysis of the beam profile monitor data [27] provides this enittance calculation. For the "profile shown in Figure 5 the emitance is $0.067 \pi \mathrm{cm} . m \mathrm{rad}$. (normulized) measured to the half width of the radinl current profile. Further measurements of beam quality are shown in Figure 6, where the beam half-width is plotted as a function of distance.

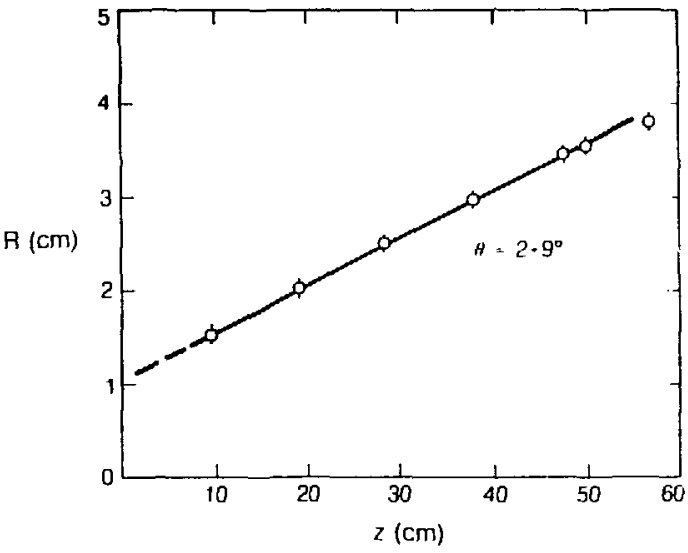

xel 8549939

Figure 6. Ream half-width va. distance from extractor.

ilere it's clear that the beam diverges linearly, that the envelope extrapulates back to the extractor radius. and thet the divergence half-angle is $50 \mathrm{mrad}$ or $2.9^{\circ}$. For the extraction voltage of $15 \mathrm{kV}$ and taking an average charge state of $3+$ for this tantalum beam, this corresponds to an emittance of $0.036 \pi$ cm.mrad.(normalized). This is close to the best emittance we've been able to obtain to-date, without collimation.

The charge state distribution of the extracted ion beam has been measured by the tume-of-night method. The spectra obtained are typically very pure, containing a low cumponent (less than a few percent) of species other than the cathode material; we can take this fortuitous behavior as evidence that the plasma originates solely ut the cathode spots, and that the trigger electrode, trigger insulator and anode da not generate 
gignificant impurities. The fit of the measured spectra to the calculated drift times is excellent. He've also messured the CSD using a magnetic analysig for some cases, and the dats obtained in this manner confirm the vime-of-fight spectra. We have noticed however that there can be some charge exchange of the beam ions after extraction.

Figure 7 shows the CSD for the case of uranium. The distribution is peaked of $\mathrm{U}^{5+}$, with sbout $40 \%$ of the total beam current in this charge atate. The CSD obtained varies with cathode muterial and with arc power. Higher $Z$ materials burn with a higher are voltage, and the average charge state is concommitantly higher also: for uranium the are voltage is up to about 100 volts, while for carbon it is about $10-15$ volts and only the gingly ionized $\mathrm{C}^{+}$is geen in the CSD. The power dissipated in the are also determines the CSD to some extent, and a plot of the average charge atate as function of are power, for tantalum, is shown in Figure 8. Note that the range of arc power spanned is about 2 - $100 \mathrm{~kW}$; for low $Z$ cathodes the power dissipated can be an order cf magnitude less.

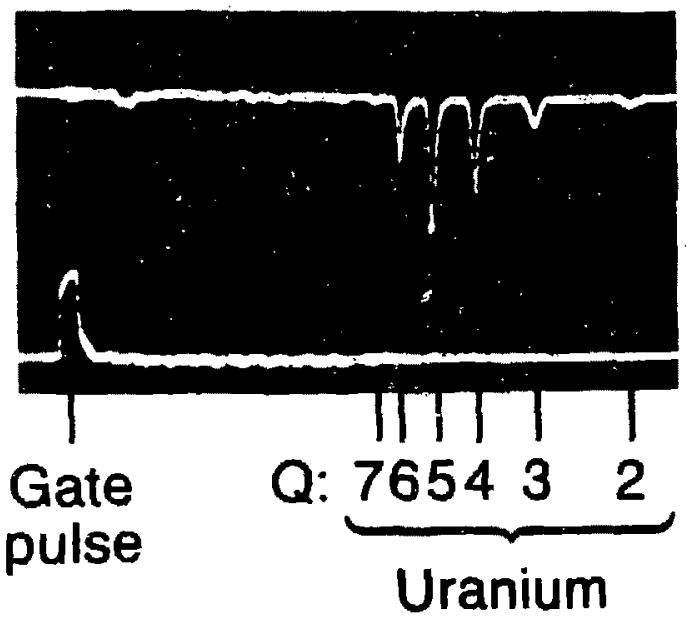

Figure 7. Uranium ion beam charge state distribution as meusured by time-of-flight diagnostic.

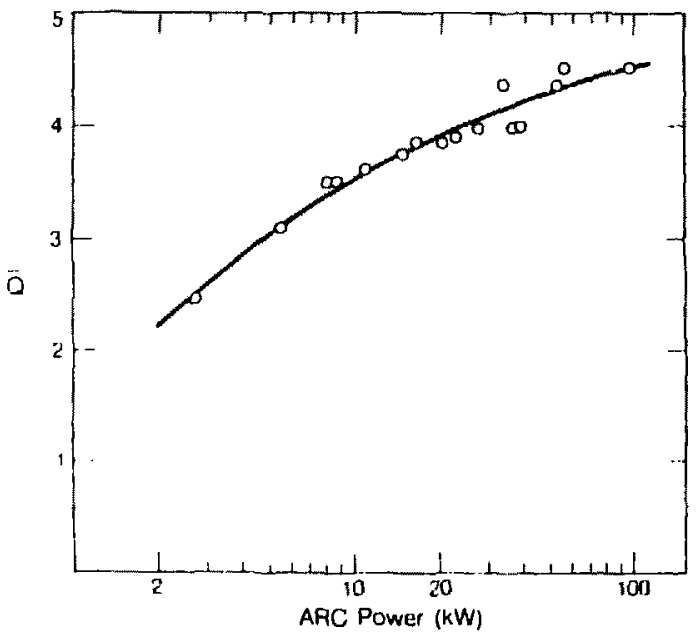

Figure 8. Mean charge state vs, arc input power, for tantolum.
It is interesting to note that a cathode fabricated from lanthanum hexaboride produces a spectrum containing both lanthanum $\left(\mathrm{La}^{2+}, \mathrm{La}^{3+}\right)$ and boron $\left(\mathrm{B}^{+}\right.$. $\left.\mathrm{B}^{2}{ }^{+}\right)$, this even though boron itself is non-metallic. One might thus generalize that the MEVVA source can produce beams containing species from non-conducting materlals when that moterial is incorporated into a conducting compound, and this might also hold for cathodes of alloyed materials.

It is important that the beam intengity and CSD remain intact throughout the entire duration of the beam pulse, and that this hold true for extraction times longer than our ubual 300 microsecond pulse. To address these concerns we've run some tests with a 3 millisecond pulse line, monitoring the time-of-night spectrs at a sequence of times throughout the pulse. In Figure 9 is shown the arc current pulse ueed and markers indicating the times at which time-of-night opectra were monitored; these spectra sre shown in Figure 10. We can make the following observations: (i). The beam CSD is similer throughout the $2.5 \mathrm{msec}$ epan monitored; (ii). The average charge state is higher earlier in the arc pulse when the arc current is higher; (iii). There is a gradual decrease in beam intensity throughout the pulse, as the are pulge line current droops. Moreover. apurt from a smoth decrease due to the falling arc current, very little jitter in the besm intensity and in CSD is seen; shot-to-shot-variation is minimal. (There is a small jitter in flight times because of imperfect extractor supply regulation).

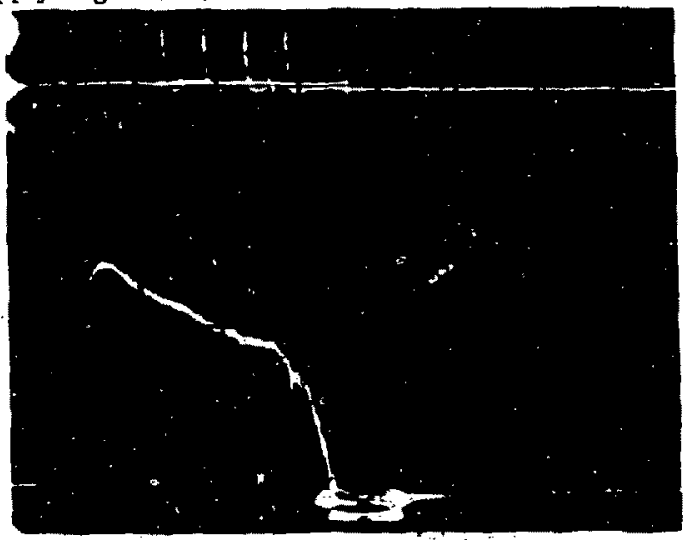

Figure 9. Long pulse arc current ind time-of-flight timing markerk. Sweep apeed $0.5 \mathrm{muec} / \mathrm{cm}$.

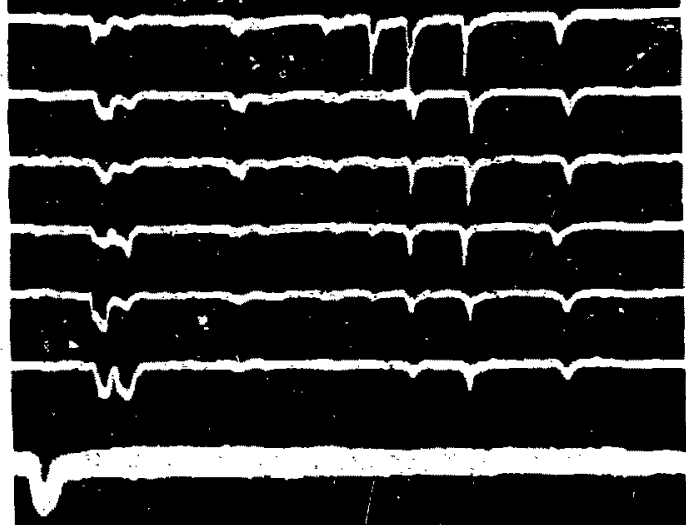

Figure 10. 'lime-ol-night CSI for the times indieated in rigure 9. Farly times top, late times bottom. 


\section{COXCLUSION}

The metal vapor vacuum arc provides a means of creating a dense plasma from a conducting solid material, from which a high current ion beam can be extracted. The source described here is one embodiment of this concept. A great deal of research and development remains to be carried out to fully characterize and understand the MEVVA ion source behavior. Improvements in design parameters will be neceagary for full exploitation of the concept. A major concern that is not so peripheral is the transport of these intense heavy ion beams in neutralization-unfriendly environments (eg, magnetic fields and accelerating columns). Given the potential of this kind of ion source, we might be confident that these developments will take place in the near future.

\section{ACKNOWLEDGRMENTS}

This work is the result of a team effort, and I am deeply indebted to Jim Galvin, Jack Gavin and Bob MacGill for a steady stresm of invaluable contributions without which this project would not have been possible.

\section{REPRRENCBS}

1. "Proceeding of the Second Symposium on Ion Sources and Formation of Ion Beams", Berkeley, CA, Oct 22-25, 1974.

2. W. B. Kunkel in "Fugion", E. Teller, ed., Academic Pres8, New York (1981).

3. S. Humphries, Jr., Nuclear Fusion 20, 1549 (1980).

4. S. Humphrieg, Jr., R. N. Sudan and L. Wiley, J. Apnl. Phyd. 47. 2382 (1976).

5. S. Humphries, Jr., C. Eichenberger and R. N. Sudan, J. Appl. Phys. 48, 2738 (1977).

6. L. Valyi, "Atom and Ion Sources", John Wiley and Sons, New York, 1977.

7. J. Ishikawg and T. Takagi, Japanege J. Appl. Phys. 22, 534 (1983).

8. J. Ishikawa, Y. Takeiri and T. Takagi, Rev. Sci. lnst rum. 55, 449 (1984).

9. A. Rockett, S. A. Barnett and J. E. Greene, J. Vac. Sci. Technol. B2, 306 (1984).
10. R. K. Feeney, W. E. Sayle, II, and J. W. Hooper, Rev. Sci. Instrum. 47. 964 (1976).

11. B. Gavin, S. Abbott, R. MacGill, R. Sorensen, J. Staples and $R$. Thatcher, IEEE Trans. Nucl. Scl. NS-28, 2684 (1981).

12. Y. Saito, Y. Mitsuoke and S. Suganomate, Rev. Sci. Ingt rum. 55, 1760 (1984).

13. S. Corley, G. Coopes, S. Humphries, Jr., M. Savage and D. Woodall, Bull. Am. Phya. Soc. 29, 1314 (1984).

14. R. J. Adler and S. T. Picraux, Nucl. Instrum, and Methode B6, 123 (1985).

15. "Vacuum Arcs - Theory and Application", J. M. Lafferty, ed., John Wiley and Sons, New York, 1980.

16. IEEE Trans. on Plasma Science, Special Issue on Vacuum Discharge Plasmas, IEEE PS-11, Sep. 1983.

17. A. S. Gllmour, Jr. and D. L. Lockwood, Proc. IEEE 60, 977 (1972).

18. J. R. Coupland, T. $S_{2}$ Green, D. P. Hammond and $A$. C. Riviere, Rev. Sci. Insrum, 44, 1258 (1973).

19. T. S. Green, IEEE Trans. Nucl. Sci. NS-23, 918 (1976).

20. J. D. Jukes, Reports on Progress in Physics 31, 333 (1968).

21. W. M. Manheimer and C. Lashmore-Davies, "MHD Instabilities in Simple Plasma Configurationan, Naval Research Laboratory, Wash. D.C., 1984.

22. J. R. Alonso, IEEE Trans. Nucl. Scl. NS-30, 1988 (1983).

23. J. R. Alonso et al. Science 217, 1135 (1982).

24. H. Gould, D. Greiner, P. Lindstrom, T. J. $M$. Symons and H. Crawford, Phys. Rev. Letterg 52, 180 (1984).

25. C. W. Kimblin, J. Appl. Phys, 44, 3074 (1973).

26. J. E. Galvin and I. G. Brown, Rev. Scl. Ingt rum. 55, 1866 (1984).

27. J. E. Galvin and I. G. Brown, Lawrence Berkeley Laboratory Report LBL-18815. (Submitted for publication in Rev. Sci. Instrum.)

\section{DISCLAIMER}

This repori was prepared as an account of work sponsored by an agency of the United States Government. Neither the United States Government nor any agency thereof, nor any of their employees, makes any warranty, express or implied, or assumes any legal liability or responsibility for the accuracy, completeness, or usefulness of any information, apparatus, product, or process disclosed, or represents that its use would not infringe privately owned rights. Reference herein to any specific commercial product, process, or service by trade name, trademark, manufacturer, or otherwise does not necessarily constitute or imply its endorsement, recommendation, or favoring by the United States Government or any agency thereof. The views and opinions of authors expressed herein do not necessarily state or reflect those of the United States Government or any agency ther cof. 